\title{
Stability and Electronic Structures of Pt-Rh Icosahedral Nanoparticles
}

\author{
Koretaka Yuge ${ }^{1}$ \\ ${ }^{1}$ Department of Materials Science and Engineering, Kyoto University, Kyoto 606-8501, Japan
}

We investigate the energetic stability and electronic structures of Pt-Rh icosahedral nanoparticles based on first-principles calculations. We find that Pt atom energetically prefers vertex and edge sites at the surface rather than subsurface and core sites, which is a tendency similar to PtRh cuboctahedral nanoparticles. This can be attributed to lower surface energy of Pt compared with Rh. Edges of nanoparticles with secondlowest coordination number are the most favorable sites for Pt atom. This could be attributed to the enhanced interatomic distance at the edge site. 1st order moment of $d$-state electronic contribution for Pt atom exhibits almost negative dependence in terms of Pt coordination number. This can be qualitatively interpreted by positive dependence of 2nd order moment of the density of states for the Pt atom on the coordination number. Pt surface segregation is expected mainly due to contribution from Pt on-site segregation energy compared with weak ordering tendency of Pt-Rh unlike-atom pairs. [doi:10.2320/matertrans.M2011055]

(Received February 15, 2011; Accepted April 6, 2011; Published May 25, 2011)

Keywords: first-principles, platinum, rhodium, alloy, nanoparticle, icosahedral, phase stability, electronic structure

\section{Introduction}

Active investigations on metal nanoparticles have been performed so far for chemical and physical applications, since they can exhibit different properties from bulk owing to high surface-to-volume ratio and finite-size effects. ${ }^{1-5)}$ Particularly, bimetallic nanoparticles are extensively investigated and synthesized in recent years in order to enhance such as catalytic properties. In order to investigate catalytic properties and thermodynamic stability for alloy nanoparticles, theoretical study based on density functional theory (DFT) has also been actively performed for Pt- and Fe-based alloys. ${ }^{6-8)}$

One of the most well-investigated systems by experiments as well as theories should be $\mathrm{Pt}-\mathrm{Rh}$ nanoparticle. There is a wide variety of experiments synthesizing the Pt-Rh nanoparticle such as $\mathrm{NaY}$-supported clusters using ion-exchange method, polyol synthesis method for clusters stabilized by ethylene glycol and $\mathrm{OH}^{-}$, pulsed laser ablation, and colloid synthesis in polymer solutions using borohydride-reduction. ${ }^{1,9-15)}$ Careful studies on their structures including atomic arrangements, size, and composition have been performed using Extended X-ray Absorption Fine Structure (EXAFS), dispersive X-ray (EDX) analysis, Transmission Electron Microscope (TEM), and X-ray photoelectron spectroscopy (XPS). Nanoparticles with diameter of 1-4 nm are typically synthesized, and their structures significantly depend on sample preparation condition or method. The Pt-Rh nanoparticles can show higher catalytic properties compared with constituent metal nanoparticle, such as $\mathrm{NO}_{x}$ reduction, $\mathrm{CO}$ chemisorption, and hydrogenation. ${ }^{15,16)}$

For Pt-Rh bulk surfaces, atomic structures and their related catalytic properties are addressed by several first-principles investigations and experiments in order to design suitable alloy surfaces that have desirable properties. Pt atoms segregate to the topmost layer and depletion at the second layer due to lower surface energy of $\mathrm{Pt}$, small mixing enthalpy, and small difference in sublimation enthalpy, which are confirmed by both previous experimental ${ }^{17-23)}$ and theoretical ${ }^{24-29)}$ investigations.
$\mathrm{Pt}$ and $\mathrm{Rh}$ metal nanoparticles have also been investigated based on DFT calculations to understand stable atomic configurations. ${ }^{30-36)} \mathrm{Up}$ to now, nanoparticles consisting of $\sim 300$ atoms are extensively studied, and a number of stable or metastable structures including icosahedron, octahedron, cuboctahedron, biplanar, and lower-symmetry shapes are proposed, while their relative stability is still under discussion.

Despite experimental as well as theoretical investigations for $\mathrm{Pt}, \mathrm{Rh}$ and $\mathrm{Pt}-\mathrm{Rh}$ nanoparticles and bulk surfaces, theoretical studies on the Pt-Rh nanoparticles have been confined to segregation profile predicted by empirical methods including free energy concentration expansion ${ }^{37)}$ and pair-bond energy model: ${ }^{38)}$ These studies predict that Pt atoms energetically prefer to segregating to the surface rather than subsurfaces. However, very recently, we investigate stability, electronic structure and quantitative segregation profile at finite temperature for $\mathrm{Pt}-\mathrm{Rh}$ cuboctahedral nanoparticles at equiatomic and Pt-dilute composition. ${ }^{39,40)} \mathrm{We}$ find that on-site segregation energy for Pt-dilute nanoparticle can be attributed to lower-order moment of the $d$-state electronic density of states, and that the segregation behavior can be qualitatively extended to Pt-Rh equiatomic nanoparticles, where multibody interaction should play significant role for quantitative estimation of segregation. With these considerations, further theoretical assessment for electronic structures and stability of different shape of nanoparticles should be highly desirable in order to essentially understand dependence of segregation behavior on the shape. In the present study, we focus on Pt-Rh icosahedral nanoparticle consisting of 55 atoms, because: (i) The 55-atom nanoparticles are considered interesting due to their intermediate size between finite molecules and fully metallic systems. ${ }^{35}$ ) (ii) Icosahedral nanoparticles are linked to cuboctahedral nanoparticles by Mackay transformation with small energy difference, and they are Platonic and Archimedean solids in uniform polyhedra. ${ }^{32)}$ (iii) The numbers of surface atoms for icosahedron and cuboctahedron are the same while the numbers of symmetry-equivalent sites and their coordination numbers are different. We perform first-principles calcula- 


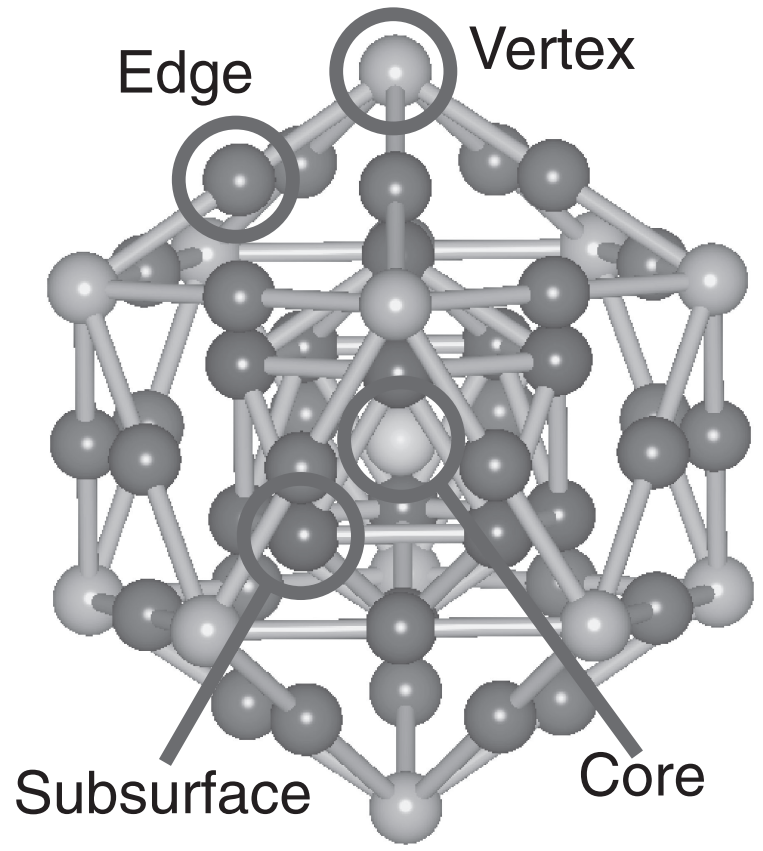

Fig. 1 Schematic illustration of 55-atom icosahedral nanoparticle used in the present calculation. Symmetry-nonequivalent four sites are shown by open circles.

tions on $\mathrm{Pt}_{1} \mathrm{Rh}_{54}$ and $\mathrm{Pt}_{2} \mathrm{Rh}_{53}$ nanoparticles with diameter of around $1 \mathrm{~nm}$. Energetically preferred single site of $\mathrm{Pt}$ atom, $\mathrm{Pt}$ electronic structures and ordering tendency for the Pt-Rh nanoparticles are discussed.

\section{Methodology}

In order to investigate energetic stability and electronic structures, we construct icosahedral nanoparticles with 55 atoms. For the size, significant size-effects can be expected since 42 of 55 atoms are at the surface. The used nanoparticle is schematically illustrated in Fig. 1. There are four symmetry-nonequivalent sites: vertex, edge, subsurface, and core. For $\mathrm{Pt}_{1} \mathrm{Rh}_{54}$ nanoparticles, we have four types of nanoparticle where single Pt atom occupies one of the four nonequivalent sites, and other 54 sites are occupied by $\mathrm{Rh}$ atoms. For $\mathrm{Pt}_{2} \mathrm{Rh}_{53}$ nanoparticles, all possible pair combinations of the four symmetry-nonequivalent sites in minimum distances are replaced by two Pt atoms, as shown in Fig. 2: There are 9 pairs.

We employ the first-principles calculations using DFT code, Vienna Ab-initio Simulation Package (VASP). ${ }^{41-43)}$ The all-electron Kohn-Sham equations are solved by employing the projector augmented-wave (PAW) method. ${ }^{44)}$ The exchange-correlation functional is described by generalized gradient approximation (GGA) of Perdew-Wang91 form. ${ }^{45)}$ Plane-wave cutoff energy of $624.8 \times 10^{-19} \mathrm{~J}(390 \mathrm{eV})$ is used throughout the calculation. The numerical errors are estimated to be of the order of $1.6 \times 10^{-22} \mathrm{~J} /$ atom $(1 \mathrm{meV} /$ atom $)$ by cutoff convergence tests. To deal with the possible convergence problems for metals, Methfessel-Paxton scheme $^{46)}$ is used with a smearing parameter $\sigma$ of $0.016 \times$ $10^{-19} \mathrm{~J}(0.01 \mathrm{eV}) . k$-points sampling is performed on the basis of the Monkhorst-Pack scheme ${ }^{47)}$ with $1 \times 1 \times 1$ grids. We put single 55-atom nanoparticle in cubic cell with each

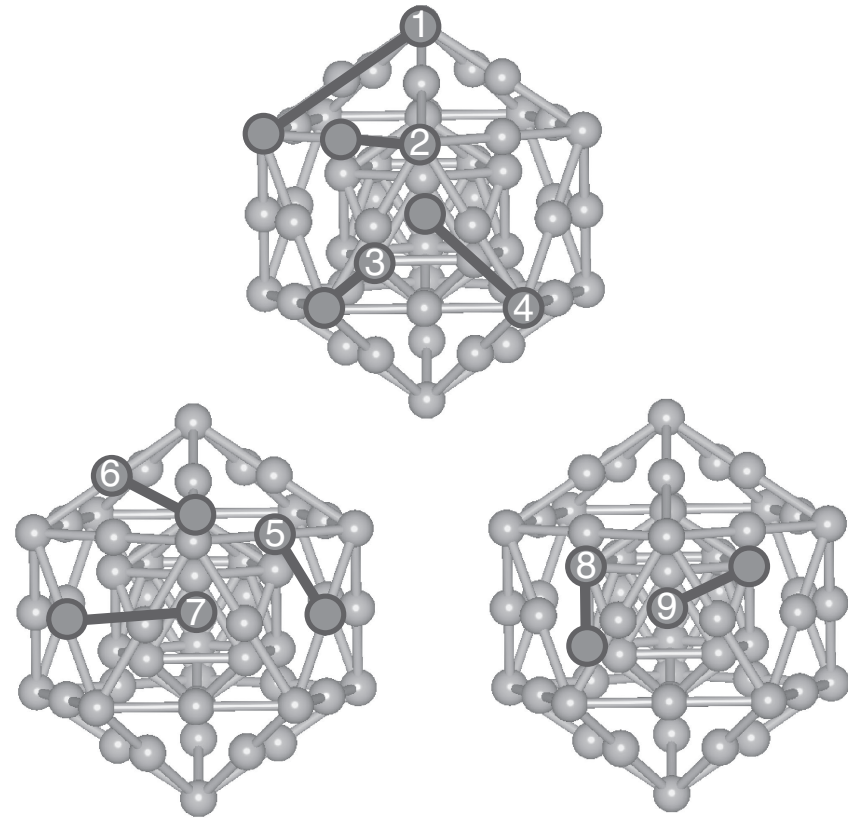

Fig. 2 Schematic illustration of positions of two $\mathrm{Pt}$ atoms in $\mathrm{Pt}_{2} \mathrm{Rh}_{53}$ nanoparticles, which are illustrated by dark spheres connected with dark bold lines.

Table 1 Calculated on-site energy of the $\mathrm{Pt}_{1} \mathrm{Rh}_{54}$ nanoparticle, $E_{\text {on-site, }}$, coordination number for Pt atom, $Z, d$-band center of Pt atoms, $\epsilon_{d}$, average of interatomic distances between $\mathrm{Pt}$ and $\mathrm{Rh}$ atoms in 1-NN coordination for the $\mathrm{Pt}_{1} \mathrm{Rh}_{54}$ nanoparticle, $\overline{\mathrm{Pt}}_{\mathrm{P}_{1} \mathrm{Rh}_{54}}$, and those between $\mathrm{Rh}$ atoms for $\mathrm{Rh}_{55}$ nanoparticle, $\bar{r}_{\mathrm{Rh}_{55}}$. In brackets, we describe estimated values for $\mathrm{Pt}_{1} \mathrm{Rh}_{54}$ cuboctahedron nanoparticles in our previous study. ${ }^{39)}$

\begin{tabular}{cccccc}
\hline Pt position & $\begin{array}{c}E_{\text {on-site }} \\
1.6 \times 10^{-19} \mathrm{~J}(\mathrm{eV})\end{array}$ & $Z$ & $\begin{array}{c}\epsilon_{d} \\
1.6 \times 10^{-19} \mathrm{~J}(\mathrm{eV})\end{array}$ & $\begin{array}{c}\bar{r}_{\mathrm{Pt}_{1} \mathrm{Rh}_{54}} \\
1.0 \times 10^{-1}\end{array}$ & $\begin{array}{c}\bar{r}_{\mathrm{Rh}_{55}} \\
\mathrm{~nm}(\AA)\end{array}$ \\
\hline vertex & $-0.87(-0.55)$ & $6(5)$ & $-2.23(-2.07)$ & 2.71 & 2.65 \\
edge & $-1.02(-0.49)$ & $8(7)$ & $-2.03(-2.14)$ & 2.74 & 2.69 \\
subsurface & $-0.21(0.07)$ & $12(12)$ & $-3.39(-3.26)$ & 2.65 & 2.64 \\
core & $0(0)$ & $12(12)$ & $-3.95(-3.35)$ & 2.58 & 2.55 \\
\hline
\end{tabular}

side of $2 \mathrm{~nm}$, which is confirmed to be sufficient in terms of cell-size dependence of the total energy. Geometry optimization under the condition of fixed cell size and shape is performed until residual forces on each atoms become less than $4.81 \times 10^{-20} \mathrm{~J} / \mathrm{nm}(0.03 \mathrm{eV} / \AA)$.

\section{Results and Discussion}

We first focus on energetics of the modeled $\mathrm{Pt}_{1} \mathrm{Rh}_{54}$ nanoparticles in order to see energetically favorable single $\mathrm{Pt}$ sites. Table 1 summarizes total energy of the nanoparticles measured from that of the nanoparticle with Pt atom at the core site (we call it on-site energy, $E_{\text {on-site, hereinafter), }}$ coordination number of $\mathrm{Pt}$ atom $(Z), d$-band center of $\mathrm{Pt}$ atom measured from the Fermi energy $\left(\epsilon_{d}\right)$, and average interatomic distances between $\mathrm{Pt}$ and $\mathrm{Rh}$ atoms in 1-NN coordination for the four modeled $\mathrm{Pt}_{1} \mathrm{Rh}_{54}$ nanoparticles, $\bar{r}_{\mathrm{Pt}_{1} \mathrm{Rh}_{54}}$, and those between $\mathrm{Rh}$ atoms in 1-NN coordination for the $\mathrm{Rh}_{55}$ nanoparticle, $\bar{r}_{\mathrm{Rh}_{55}}$. In brackets, we describe value for $\mathrm{Pt}_{1} \mathrm{Rh}_{54}$ cuboctahedron nanoparticles obtained in our previous study. ${ }^{39)}$ From Table 1 , we can clearly see the 
significant negative value of on-site energy for vertex and edge sites at the surface. For bulk, Pt atoms segregate to the surface due to negative on-site energy mainly coming from lower Pt surface energy compared with $\mathrm{Rh}^{27-29)}$ For the $\mathrm{Pt}$ and Rh nanoparticles, we calculate surface energy defined by

$$
\Delta E_{\text {surf }}=\frac{E_{\text {icosa }}-N_{\text {icosa }} E_{\text {bulk }}}{N_{\text {surf }}}
$$

where $E_{\text {icosa }}$ denotes total energy of $\mathrm{Pt}$ or $\mathrm{Rh}$ icosahedral nanoparticle with 55 atoms, $N_{\text {icosa }}$ the number of atoms in the nanoparticle, $E_{\text {bulk }}$ total energy of bulk Pt or Rh per atom, and $N_{\text {surf }}$ the number of surface atoms in the nanoparticle. Estimated surface energy of $\mathrm{Pt}\left(E_{\text {surf }}^{\mathrm{Pt}}=2.15 \times 10^{-19} \mathrm{~J} /\right.$ atom $(1.34 \mathrm{eV} /$ atom $))$ is smaller than that of $\mathrm{Rh}\left(E_{\text {surf }}^{\mathrm{Rh}}=2.50 \times\right.$ $10^{-19} \mathrm{~J} /$ atom $(1.56 \mathrm{eV} /$ atom $)$ ). Therefore, when we assume weak mixing contribution to the total free energy, which holds for the bulk Pt-Rh alloy as well as the Pt-Rh cuboctahedral nanoparticle, Pt surface segregation is expected for the icosahedral nanoparticles due to lower Pt surface energy of the nanoparticle. Another important point is that for icosahedron, on-site energies of vertex and edge sites are around two times larger in negative sign than those for cuboctahedron. Therefore, compared to the cuboctahedron, more pronounced Pt surface segregation is expected for the icosahedron. At the surface, on-site energy increases in negative sign when coordination number of $Z$ decreases. This can be attributed to the fact that Pt atoms segregate to sites with lower coordination number in order to decrease surface energy of the nanoparticle in terms of effective medium approximation up to 2 nd order moment. ${ }^{39)}$ This tendency perfectly holds for cuboctahedral nanoparticles as described in Table 1, while it has an exception for icosahedron: Edge sites with higher coordination number of $Z=8$ have larger on-site energy in negative sign than vertex sites with lower coordination number of $Z=6$. In order to interpret this, we exhibit average interatomic distances for the $\mathrm{Pt}_{1} \mathrm{Rh}_{54}$ and $\mathrm{Rh}_{55}$ nanoparticles as described in Table 1. For all four sites, interatomic distances of the $\mathrm{Pt}_{1} \mathrm{Rh}_{54}$ nanoparticles are respectively larger than those of $\mathrm{Rh}_{55}$, which can be naturally attributed to larger atomic radius of $\mathrm{Pt}$ atom than that of $\mathrm{Rh}$. Interatomic distances tend to increase with decrease in coordination number, while for edge sites, average interatomic distances are $0.274 \mathrm{~nm}\left(2.74 \AA\right.$ ) for $\mathrm{Pt}_{1} \mathrm{Rh}_{54}$ and $0.269 \mathrm{~nm}(2.69 \AA)$ for $\mathrm{Rh}_{55}$ nanoparticle, which are respectively larger than those for vertex sites of $0.271 \mathrm{~nm}(2.71 \AA)$ and $0.265 \mathrm{~nm}(2.65 \AA)$. Therefore, such enhanced interatomic distances for the edge sites should contribute to reversal onsite energy between edge and vertex sites because of (i) larger atomic radius of $\mathrm{Pt}$ than $\mathrm{Rh}$ preferring edge sites with larger spacing, and (ii) decrease in actual coordination number of edge sites.

Next, we focus on the electronic structures of Pt atom in the nanoparticles. Figure 3 shows the calculated electronic density of states from $d$-state contribution measured from the Fermi energy for the two surface sites. From Fig. 3, no significant difference can be found for the two surface sites. Corresponding $d$-band center, $\epsilon_{d}$, is described in Table 1 . $\epsilon_{d}$ for sites at the surface ranges from $-3.57 \times 10^{-19}$ to $-3.25 \times 10^{-19} \mathrm{~J}(-2.23$ to $-2.03 \mathrm{eV})$, which is larger in negative sign than $\epsilon_{d}=-3.24 \times 10^{-19} \mathrm{~J}(-2.02 \mathrm{eV})$ for the

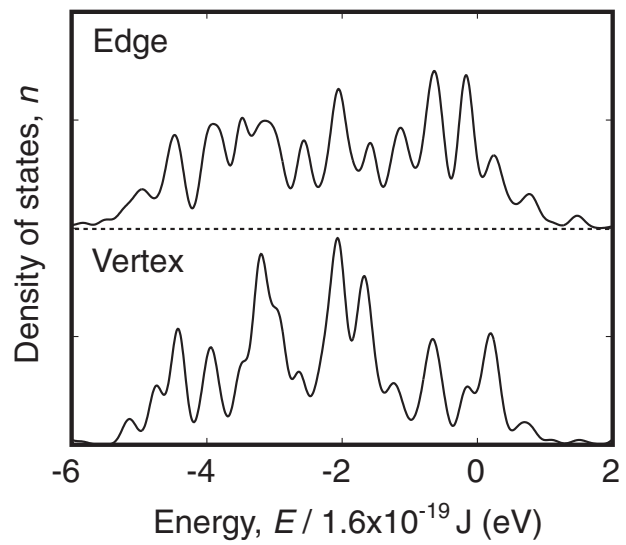

Fig. 3 Calculated density of states for the Pt $d$-band measured from the Fermi energy for vertex and edge sites at the surface.

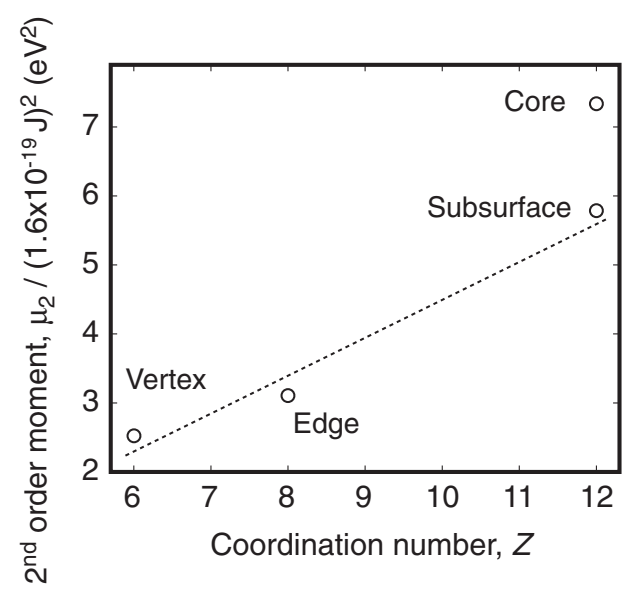

Fig. 4 2nd order moment of the Pt $d$-band, as a function of coordination number for the Pt atom. Dashed line guides the eyes for vertex, edge, and subsurface sites.

$\mathrm{Pt}_{25} \mathrm{Rh}_{75}$ ground-state bulk alloy surface. ${ }^{29)}$ Another important point is that $\epsilon_{d} \mathrm{~S}$ of sites at the surface are shallower than those of subsurface and core sites with larger coordination number, which is a tendency similar to cuboctahedral nanoparticles. This can be naturally attributed to increase in 2nd order moment of the $\mathrm{Pt} d$-band with increase in coordination number, since $\mathrm{Pt}$ has more than half-filled $5 d$ shells. In order to intuitively see this, we show in Fig. 4 the 2 nd order moment of $\mathrm{Pt} d$-band with respect to the $d$-band center as a function of coordination number for the Pt atom. We can see from Fig. 4 that with increase in coordination number, 2nd order moment certainly increases, which should lead to downshift of the corresponding Pt $d$-band center at subsurface and core sites. However, 2nd order moment does not seem to have linear dependence on coordination number. This is because of difference in coordination circumstances for symmetry-distinct four sites: For the core site, neighboring $\mathrm{Rh}$ atoms are in icosahedral shape, while for vertex, edge, and subsurface sites, they are in combination of pentagonal pyramid and prism. Therefore, we draw the broken line in Fig. 4 to guiding the eyes for vertex, edge and subsurface sites with the same type of coordination circumstances. In this case, we can see almost linear dependence of 2 nd order moment for the vertex, edge and subsurface sites. Slight 


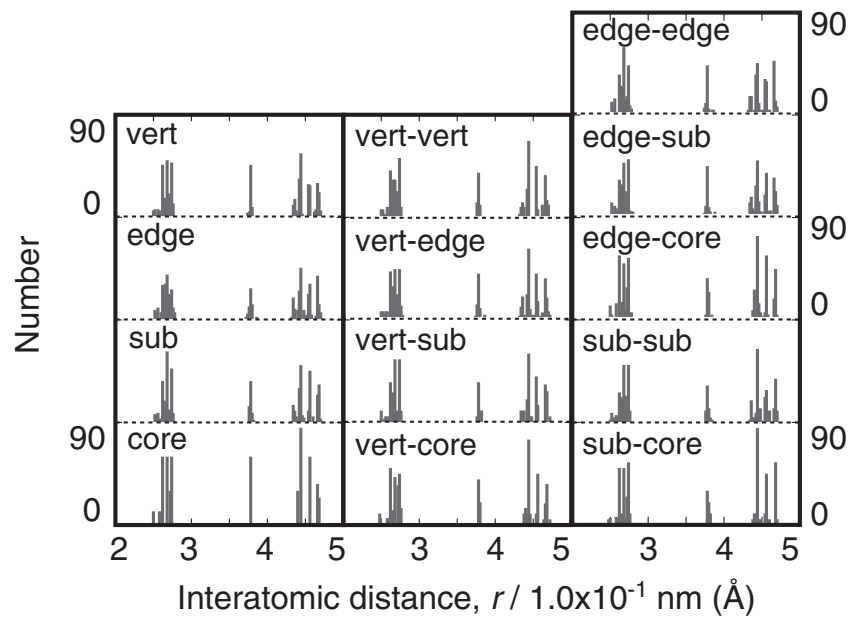

Fig. 5 Distribution of interatomic distances up to the 3-NN coordination for $\mathrm{Pt}_{1} \mathrm{Rh}_{54}$ (left-hand row) and $\mathrm{Pt}_{2} \mathrm{Rh}_{53}$ (middle- and right-hand rows) nanoparticles.

negative deviation from the broken line for the edge sites would be partially attributed to the enhanced increase in interatomic distances between Pt and neighboring Rh atoms at edge sites, which is already discussed above.

Finally, we qualitatively investigate ordering tendency for the icosahedral nanoparticles. In order to understand ordering tendency including energetic preference of $\mathrm{Pt}$ atoms, we introduce Pt-Pt pair interaction energy, $E_{\mathrm{I}-\mathrm{J}}$, defined by our previous work. ${ }^{39)}$ Here, $E_{\mathrm{I}-\mathrm{J}}<0$ indicates preference of $\mathrm{Pt}$ pair at $\mathrm{I}$ and $\mathrm{J}$ sites, and $E_{\mathrm{I}-\mathrm{J}}>0$ disfavor the Pt-Pt pair. We should note here that in order to apply the defined pair interaction energies to interpreting preference of individual atomic pairs, it would be required that structural differences between $\mathrm{Pt}_{1} \mathrm{Rh}_{54}$ and $\mathrm{Pt}_{2} \mathrm{Rh}_{53}$ nanoparticles can be small. In order to confirm the structural differences, we show in Fig. 5 distribution of interatomic distances up to 3-NN coordination for $\mathrm{Pt}_{1} \mathrm{Rh}_{54}$ (left-hand row) and $\mathrm{Pt}_{2} \mathrm{Rh}_{53}$ (middle- and righthand rows) nanoparticles. For all the nanoparticles, we can see the following similar tendencies for the distribution of interatomic distances, which certainly imply small structural differences between $\mathrm{Pt}_{1} \mathrm{Rh}_{54}$ and $\mathrm{Pt}_{2} \mathrm{Rh}_{53}$ nanoparticles: (i) There are three characteristic distributions with interatomic distances of around $0.26 \mathrm{~nm}(2.6 \AA), 0.37 \mathrm{~nm}(3.7 \AA)$, and $0.45 \mathrm{~nm}(4.5 \AA)$, which respectively correspond to pairs in $1-\mathrm{NN}, 2-\mathrm{NN}$, and 3-NN. (ii) Pairs in 1-NN and 3-NN have three characteristic interatomic distances respectively, and (iii) Pairs in 2-NN have single characteristic interatomic distance, and have narrower distribution than those in 1-NN and 3-NN. Figure 6 shows interaction energies for the Pt pairs shown in Fig. 2. Most of the interaction energies (8 of 9) exhibit positive sign with larger value compared with that in negative sign, indicating that Pt-Pt pairs are energetically unfavored and Pt-Rh unlike-atom pairs are favored in the Pt$\mathrm{Rh}$ nanoparticles, which is an ordering tendency similar to the bulk Pt-Rh alloy as well as the Pt-Rh cuboctahedral nanoparticle. ${ }^{29,39)}$ Another important point in Fig. 6 is that $E_{\mathrm{I}-\mathrm{J} S}$ are in one-order smaller than $\mathrm{Pt}$ on-site energies described in Table 1. This indicates that $\mathrm{Pt}$ on-site energies should be dominant and ordering tendency is predominant contributions to surface segregation in the $\mathrm{Pt}-\mathrm{Rh}$ nano-

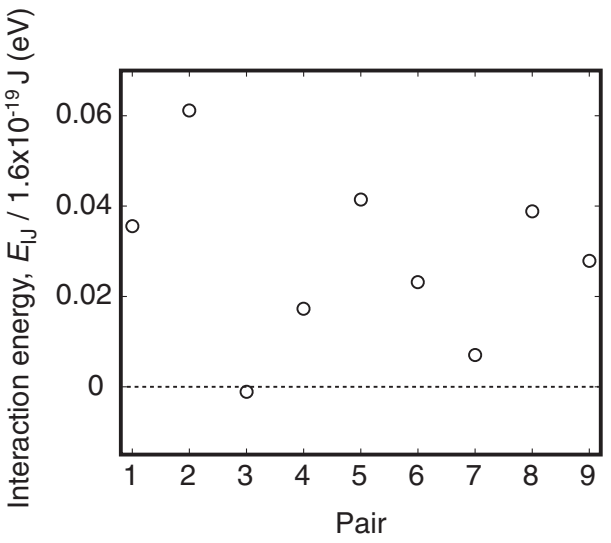

Fig. 6 Calculated Pt-Pt interaction energy for the pairs shown in Fig. 2.

particles. Therefore, Pt-Rh icosahedral nanoparticles are expected to show segregation behavior similar to the Pt-Rh bulk surface and the Pt-Rh cuboctahedral nanoparticle, where strong Pt segregation is mainly attributed to Pt on-site energy contribution. ${ }^{29,39)}$

\section{Conclusions}

First-principles calculations are employed in order to examine energetic stability and electronic structures of Pt-Rh icosahedral nanoparticles. Single Pt atom energetically prefers vertex and edge sites at the surface rather than subsurface and core sites. This can be attributed to the fact that $\mathrm{Pt}$ tends to prefer sites with lower coordination number so that surface energy of the nanoparticle decreases, which has also been seen in Pt-Rh bulk alloy surfaces as well as cuboctahedral nanoparticles. However, unlike cuboctahedron, edge sites with larger coordination number have larger on-site energy compared with vertex sites with the smallest coordination number. This can be partially attributed to the enhanced interatomic distances at the edge sites. $d$ band center for Pt atom exhibits almost negative dependence with respect to Pt coordination number. This can be attributed to an increase in 2nd order moment of the Pt $d$-band with an increase in the coordination number. Ordering tendency for the $\mathrm{Pt}-\mathrm{Rh}$ nanoparticles is also investigated. Pt-Rh unlikeatom pairs are energetically preferred, which is a tendency similar to the Pt-Rh bulk alloy and the Pt-Rh cuboctahedral nanoparticle. Pt segregation to the surface of Pt-Rh nanoparticle is expected due to the dominant contribution of Pt onsite energy compared with weak ordering tendency.

\section{Acknowledgements}

This research was supported by Grant-in-Aid for Young Scientists B (22760502) by JSPS.

\section{REFERENCES}

1) N. Toshima and T. Yonezawa: New J. Chem. 1 (1998) 1179-1201.

2) M. Valden, X. Lai and D. W. Goodman: Science 281 (1998) 16471650 .

3) J. Meier, K. A. Friedrich and U. Stimming: Faraday Discuss. 121 (2002) 365-372. 
4) N. Lopez and J. K. Norskov: J. Am. Chem. Soc. 124 (2002) 11262 11263.

5) F. Maillard, M. Eikerling, O. V. Cherstiouk, S. Schreier, E. Savinova and U. Stimming: Faraday Discuss. 125 (2004) 357-377.

6) Y. Wang, G. M. Stocks, A. Rusanu, D. M. C. Nicholson, M. Eisenbach, Q. Zhang and J. P. Liu: IEEE Trans. Mag. 43 (2007) 3103-3105.

7) A. A. Franco, S. Passot, P. Fugier, C. Anglade, E. Billy, L. Guétaz, N. Guillet, E. De Vito and S. Mailley: J. Electrochem. Soc. 156 (2009) B410-424.

8) M. E. Gruner and A. Dannenberg: J. Mag. Mag. Mater. 321 (2009) 861-864.

9) N. Savastenko, H. R. Volpp, O. Gerlach and W. Strehlau: J. Nanopart Res. 10 (2008) 277-287.

10) Y. Wang, J. Zhang, X. Wang, J. Ren, B. Zuo and Y. Tang: Topics Catal. 35 (2005) 35-41.

11) M. Harada, K. Asakura and N. Toshima: J. Phys. Chem. 98 (1994) 2653-2662.

12) T. Hashimoto, K. Saijo, M. Harada and N. Toshima: J. Chem. Phys. 109 (1998) 5627-5638.

13) M. Harada and H. Einaga: J. Colloid Int. Sci. 308 (2007) 568-572.

14) E. Cimini and R. Prins: J. Phys. Chem. B 101 (1997) 5285-5293.

15) K. Siepen, H. Bönnemann, W. Brijoux, J. Rothe and J. Hormes: Appl. Organometal. Chem. 14 (2000) 549-556.

16) C. E. Lyman, R. E. Lakis and H. G. Stenger: Ultramicroscopy 58 (1995) 25-34.

17) F. C. M. J. M. van Delft and B. E. Nieuwenhuys: Surf. Sci. 162 (1985) $538-543$.

18) A. D. van Langeveld and J. W. Niementsverdriet: Surf. Sci. 178 (1986) 880-887.

19) D. M. Ren and T. T. Tsong: Surf. Sci. 184 (1987) L439-L444.

20) P. Verga and M. Schmid: Appl. Surf. Sci. 141 (1999) 287-293.

21) E. Platzgummer, M. Sporn, R. Koller, S. Forsthuber, M. Schmid, W. Hofer and P. Varga: Surf. Sci. 419 (1999) 236-248.

22) E. L. D. Hebenstreit, W. Hebenstreit, M. Schmid and P. Varga: Surf. Sci. 441 (1999) 441-453.
23) D. Brown, P. D. Quinn, D. P. Woodruff, T. C. Q. Noakes and P. Bailey: Surf. Sci. 497 (2002) 1-12.

24) B. Legrand and G. Tréglia: Surf. Sci. 236 (1990) 398-408.

25) J. Florencio, D. M. Ren and T. T. Tsong: Surf. Sci. 245 (1996) L29L33.

26) L. Z. Mezey and W. Hofer: Surf. Sci. 402-404 (1998) 845-850.

27) A. V. Ruban and H. L. Skriver: Comput. Mater. Sci. 15 (1999) 119_ 143.

28) S. Müller, M. Stöhr and O. Wieckhorst: Appl. Phys. A 82 (2006) 415419.

29) K. Yuge, A. Seko, A. Kuwabara, F. Oba and I. Tanaka: Phys. Rev. B 74 (2006) 174202 1-13.

30) V. Kumar and Y. Kawazoe: Phys. Rev. B 77 (2008) 205418 1-10.

31) S. K. Nayak, S. E. Weber, P. Jena, K. Wildberger, R. Zeller, P. H. Dederichs, V. S. Stepanyuk and W. Hergert: Phys. Rev. B 56 (1997) 8849-8854.

32) C. Barreteau, M. C. Desjonquëres and D. Spanjaard: Eur. Phys. J. D 11 (2000) 395-402.

33) L. Wang and Q. Ge: Chem. Phys. Lett. 366 (2002) 368-376.

34) V. Kumar and Y. Kawazoe: Eur. Phys. J. D 24 (2003) 81-84

35) E. Aprà and A. Fortunelli: J. Phys. Chem. 107 (2003) 2934-2942.

36) L.-L. Wang and D. D. Johnson: Phys. Rev. B 75 (2007) 235405 1-10.

37) R. Vardi, L. Rubinovich and M. Polak: Surf. Sci. 602 (2008) 10401044.

38) A. de Sarkar and B. C. Khanra: Chem. Phys. Lett. 353 (2002) 426-430.

39) K. Yuge, T. Ichikawa and J. Kawai: Mater. Trans. 51 (2010) 321-324.

40) K. Yuge: J. Phys.: Condens. Matter 22 (2010) 245401 1-6.

41) G. Kresse and J. Hafner: Phys. Rev. B 47 (1993) R558-561.

42) G. Kresse and J. Furthmüller: Phys. Rev. B 54 (1996) 11169-11186.

43) G. Kresse and D. Joubert: Phys. Rev. B 59 (1999) 1758-1775.

44) P. E. Blöchl: Phys. Rev. B 50 (1994) 17953-17979.

45) J. P. Perdew and Y. Wang: Phys. Rev. B 45 (1992) 13244-13249.

46) M. Methfessel and A. T. Paxton: Phys. Rev. B 40 (1989) 3616-3621.

47) H. J. Monkhost and J. D. Pack: Phys. Rev. B 13 (1976) 5188-5192. 